\title{
Development and preliminary validation of the physician support of skin self-examination scale
}

\author{
Adina Coroiu ${ }^{1}$, Chelsea Moran ${ }^{1}$, Rosalind Garland ${ }^{2}$ and Annett Körner ${ }^{1}$ \\ ${ }^{1}$ Department of Educational and Counselling Psychology, McGill University, Montreal, Québec, Canada \\ ${ }^{2}$ Ingram School of Nursing, McGill University, Montréal, Québec, Canada
}

\begin{abstract}
Skin self-examination (SSE) is a crucial preventive health behaviour in melanoma survivors, as it facilitates early detection. Physician endorsement of SSE is important for the initiation and maintenance of this behaviour. This study focussed on the preliminary validation of a new nine-item measure assessing physician support of SSE in melanoma patients. English and French versions of this measure were administered to 188 patients diagnosed with melanoma in the context of a longitudinal study investigating predictors and facilitators of SSE. Structural validity was investigated using exploratory factor analysis conducted in Mplus and convergent and divergent validity was assessed using bivariate correlations conducted in spss. Results suggest that the scale is a unidimensional and reliable measure of physician support for SSE. Given the uncertainty regarding the optimal frequency of SSE for at-risk individuals, we recommend that future psychometric evaluations of this scale consider tailoring items according to the most up-to-date research on SSE effectiveness.
\end{abstract}

Key words: melanoma, skin self examination (SSE), physician support of SSE

Received 7 March 2017; revised 13 September 2017; accepted 16 September 2017; first published online 17 October 2017

Physician's support and endorsement of preventive health behaviours are important predictors of adherence to medical regimens among individuals diagnosed with cancer (Bellizzi et al., 2005). Common reactions to a melanoma diagnosis, both immediate and during the 10-year follow-up period, include 'trusting my doctors' and 'following the medical advice exactly' (Zschocke et al., 1996). Melanoma survivors have a ninefold increased risk for developing a subsequent primary melanoma compared to the general population (Bradford et al., 2010). Most practice guidelines for follow-up care recommend skin self-examination (SSE) for melanoma survivors (Marciano et al., 2014; Watts et al., 2015), as a means of facilitating early detection and timely treatment. Early detection is a key contributor to reductions in melanoma-related mortality (Katalinic et al., 2012). Research has found that upwards

Correspondence to: Adina Coroiu, MA, PhD Candidate, Department of Educational and Counselling Psychology, McGill University, Room 614, 3700 McTavish Street, Montréal, Québec, Canada H3A 1Y2. Email: acoroiu@gmail.com

(C) Cambridge University Press 2017
$61 \%$ of melanomas are self-detected as opposed to physician-detected (Carli et al., 2003; Geller, 2009), and those who practice SSE are diagnosed with thinner (earlier disease) melanoma compared to those who do not (Carli et al., 2003; Swetter et al., 2012). Further, analyses from a recent randomized controlled trial (Robinson et al., 2016) found that over 24 months, melanoma survivors who had been instructed to check their skin identified 43 new melanomas, whereas survivors who had not specifically been trained or instructed to check their skin did not identify any new melanomas. Among melanoma survivors, reported rates for thorough (entire body) and regular SSE (at least every two months) range from 14 to 39\% (Loescher et al., 2006; Manne and Lessin, 2006; Mujumdar et al., 2009; Pollitt et al., 2009; Coups et al., 2016).

Physician recommendation for SSE and live demonstration of how to inspect the skin for problematic lesions play a crucial role in the initiation and maintenance of SSE. For example, individuals with a personal history of melanoma who have been instructed or shown how to check their skin by 
physicians rated this behaviour as more important (Zschocke et al., 2000) and were more likely to perform SSE compared to those who did not receive such instructions (Manne et al., 2004; Coups et al., 2016). Currently, it is unknown what the rates for physician recommendation of SSE to newly diagnosed patients are across medical settings. A small pilot conducted by the current authors in a melanoma clinic in Quebec, Canada found that among 41 newly diagnosed patients, 71\% $(n=29)$ reported that a physician had recommended they perform regular SSE post diagnosis compared to only $37 \%(n=15)$ who reported receiving similar recommendations prior to diagnosis (Körner et al., 2013a). In another study conducted in the United States with melanoma survivors $(n=177), 37 \%$ of patients reported having been shown by a healthcare professional how to correctly perform SSE, but the rates of healthcare professional recommendations for SSE were not reported (Coups et al., 2016). Failure to recommend SSE to patients at high-risk is a missed opportunity for skin cancer prevention at the level of primary healthcare, with important and potentially devastating ramifications for patients and their families, as well as the medical system, as substantially more resources are allocated to managing metastatic melanoma versus earlier stages (Tsao et al., 1998).

Studies conducted with melanoma patients typically use patient-reported outcomes to assess whether physicians recommended SSE. As no standardized self-report measure of physician support of SSE currently exists, our team developed a measure based on clinical practice guidelines for melanoma follow-up care (for reviews of clinical guidelines, see Marciano et al., 2014; Watts et al., 2015), which stress the importance of physician recommendation for SSE and demonstration of SSE. The aim of the current study was to report on the preliminary validation of the Physician Support of SSE Scale, including factorial structure, convergent and divergent validity, and internal consistency reliability.

\section{Method}

\section{Participants and procedures}

This study is a secondary analysis using baseline data collected for a longitudinal study investigating barriers and facilitators of SSE among melanoma survivors. English and French-speaking participants were recruited from two hospitals in Montréal, Canada between 2012 and 2016. Participants completed self-report baseline questionnaires assessing socio-demographic information and psychosocial constructs, including physician's recommendation and support of SSE, patient distress, intention to perform SSE, and self-efficacy for SSE. Patient health information was extracted from hospital medical charts. Institutional Research Boards of McGill University and participating hospitals approved the study.

\section{Measures}

Development of the Physician Support of SSE Scale A large pool of items, using different phrasing in English, was created to assess physician support of SSE. When choosing the item content, we consulted clinical care guidelines, which suggest that clinicians should recommend SSE to high-risk individuals, but also show how SSE should be performed, and also our pilot data from a study with non-melanoma skin cancer. The final version of the scale included nine items, which encompass various aspects of SSE-related support provided by physicians, such as pragmatic (cognitive) aspects ('My physician has asked me if I have questions or concerns about examining my skin'), behavioural components ('My physician has pointed out a lesion(s) that I should keep an eye on'), and perceptions of physician attitudes towards SSE ('It seems important to my physician that I do skin selfexams'). In addition to recommendations to perform SSE, we also included items assessing recommendations for regular SSE and SSE of the entire body. This is a self-report scale to be completed by patients: patients were instructed to relay their experience with one physician involved in their melanoma care in the previous three months. To minimize recall bias, the items were scored on a Likert scale ranging from 0 (not at all true) to 3 (true) as opposed to a dichotomous scale, which is often used for rating observable behaviours. The questionnaire was developed in English and then translated into Canadian French to reflect the language needs of our Québécois patients. An authorized French translator with prior experience in health research translated the English items into French (forward translation) and one bilingual research assistant who identified as Anglophone (ie, native English speaker) translated the French items back into English (backward translation). 
Subsequently, two bilingual members of our research group evaluated the forward and backward translations against each other and suggested changes, which were discussed with the French authorized translator, who then finalized the French version. Two independent bilingual reviewers familiar with psychosocial oncology research, and one community member (ie, cancer patient) ensured the clarity and relevance of the items and approved the final English and French versions.

Physician support of SSE was assessed via the newly developed nine-item scale, with responses ranging from 0 (not at all true) to 3 (true), and possible scores between 0 and 27 .

Additional measures used for the validation of the Physician Support of SSE Scale

The 15-item Health Care Climate Questionnaire (HCCQ; Williams et al., 1996) was used to assess autonomy-supportive attitudes from healthcare providers. The HCCQ responses range from 1 (strongly disagree) to 7 (strongly agree) with possible scores between 15 and 105. The HCCQ is commonly used to measure perceived healthcare provider support in samples of patients with chronic illness, which showed good psychometric properties across multiple samples (Williams et al., 1996). The 15-item Skin Cancer Index (SCI) (Rhee et al., 2005) was used to assess skin cancerspecific distress. The SCI responses range from 1 (very much) to 5 (not at all), with possible scores between 15 and 75. Intentions to perform SSE were assessed using one item, 'How likely are you to self-examine your skin on a regular basis in the coming year?' scored on a range from 1 (very unlikely) to 5 (very likely). Frequency of clinical skin exams, recommendations for SSE, and demonstration of SSE by healthcare professional prior to current melanoma diagnosis (Körner et al., 2013b) were assessed using three individual items scored on a scale ranging from 0 (never) to 3 (a few times a year). For all measures, higher scores indicated more of the measured construct, with items of the SCI being reverse coded to reflect this trend.

\section{Data analysis plan}

Analyses were conducted with patients diagnosed within five years of study participation, given the more frequent follow-up regimen during this time period at our recruiting hospitals (Wang, 2007). Exploratory Factor Analysis (EFA) with weighted least squares parameter estimation and oblique rotation was conducted with the nine-item Physician Support of SSE Scale. As per recommended guidelines for assessing model fit (Hu and Bentler, 1999), in addition to the $\chi^{2}$ test, which is highly sensitive to sample size and potentially leading to erroneous rejection of the model fit (Reise et al., 1993), we used a combination of other model fit indices: the Tucker-Lewis index (TLI; Tucker and Lewis, 1973), the comparative fit index (CFI; Bentler, 1990) and the root mean square error of approximation (RMSEA; Steiger, 1990). Good fitting models are indicated by a TLI and CFI $\geqslant 0.95$ and RMSEA $\leqslant$ 0.06 (Hu and Bentler, 1999), although a CFI and TLI of 0.90 or above (Kline, 2005) and a RMSEA of 0.08 or less (Browne and Cudeck, 1993) are regarded as indicators of an adequate model fit. These analyses were conducted using Mplus 7 (Muthén and Muthén, 2007), which is a modelling software preferred for factor analysis over SPSS.

Descriptive statistics were computed for the study sample. Cronbach's $\alpha$ was computed for the Physician Support of SSE scale. Convergent and divergent validity of the scale were assessed through Pearson's and Spearman correlations with socio-demographic (age, education) and medical (cancer stage) variables and the study measures. The magnitude of the bivariate correlations was interpreted following Cohen's effect size descriptors, that is, $r \leq 0.10$ indicating small, $r=0.30$ indicating moderate, and $r=0.50$ indicating large differences (Cohen, 1988). These analyses were conducted using SPSS, version 20.

\section{Results}

\section{Sample characteristics}

Current secondary analysis included patients diagnosed with melanoma within past five years $(n=188 ; 78 \%$ of our total sample). Sample characteristics are included in Table 1.

\section{Factorial structure of the Physician Support of SSE Scale}

An EFA with the nine-item scale showed less than ideal, but acceptable fit, $\chi^{2}(n=188, \mathrm{df}=27)=85.61$, $P=0.000$, RMSEA $=0.11,90 \%$ CI [0.08, 0.13], Primary Health Care Research \& Development 2018; 19: 301-308 
Table 1 Sample characteristics $(n=188)$

\begin{tabular}{lcc}
\hline Variables & $\%(n)$ & M (SD) \\
\hline Gender & & \\
$\quad$ Female & $49(92)$ & \\
Male & $51(95)$ & $59(14)$ \\
Age (in years) & & $15(3.5)$ \\
Education level (in years) & $79(148)$ & \\
Lived in Canada entire life & & \\
Income & $1(2)$ & \\
$\quad$ 10 000 & $4(8)$ & \\
10-19999 & $5(10)$ & \\
20-29999 & $4(8)$ & \\
$30-39999$ & $10(19)$ & \\
$40-59999$ & $21(39)$ & \\
$60-79999$ & $35(66)$ & \\
>80 000 & $19(36)$ & \\
Missing & & \\
Melanoma stage at diagnosis & $18(33)$ & \\
0 (in situ) & $50(93)$ & \\
I & $22(42)$ & \\
II & $6(11)$ & \\
III & $4(8)$ & \\
IV & & \\
Missing & & \\
HCCQ & & \\
SCl & & \\
Intention to perform SSE & & \\
Physician support for SSE & & \\
& & \\
\hline
\end{tabular}

$\mathrm{HCCQ}=$ Health Care Climate Questionnaire; $\mathrm{SCI}=$ Skin Cancer Index; SSE = skin self-examination.

$\mathrm{CFI}=0.998, \mathrm{TLI}=0.997$. As a sensitivity analysis, we conducted EFA's with the French $\left(\chi^{2}(n=94\right.$, $\mathrm{df}=27)=51.00, \quad P=0.004, \quad \mathrm{RMSEA}=0.10, \quad 90 \%$ CI $[0.06,0.14], \quad$ CFI $=0.997, \quad$ TLI $=0.996)$ and English $\quad\left(\chi^{2}(n=94, \quad \mathrm{df}=27)=47.83, \quad P=0.000\right.$, RMSEA $=.009,90 \%$ CI [0.05, 0.13], CFI $=0.999$, $\mathrm{TLI}=0.998$ ) subsamples and found similar results.

Detailed statistics for the nine-item scale, including factor loadings and inter-item correlations computed across the entire sample, were included in Table 2 . We noted high $(\geqslant 0.9)$ interitem correlations between item \#4 ('My physician has recommended that I do skin exams') and item \#5 ('My physician has recommended that I examine my skin regularly'), and between item \#5 and item \#6 ('My physician has recommended that I examine the skin of my whole body'). A second EFA excluding item \#5 showed acceptable fit, $\chi^{2}(n=188, \quad \mathrm{df}=20)=68.75$, $P=0.000$, RMSEA $=0.11,90 \%$ CI $[0.09,0.14]$, $\mathrm{CFI}=0.996$, TLI $=0.995)$.

Primary Health Care Research \& Development 2018; 19: 301-308

\section{Validity and reliability of the nine-item Physician Support of SSE scale}

There were no associations with demographic information, such as age, education, and with medical variables, melanoma stage, time since diagnosis. The Physician Support for SSE Scale was positively associated with the HCCQ $(r=0.39$, $P<0.001$ ), prior healthcare professional (HCP) recommendations for SSE $(r=0.36, P<0.001)$, prior HCP-led skin exam $(r=0.18, P=0.014)$, prior HCP demonstration of SSE $(r=0.26$, $P<0.001)$, and intention to perform SSE $(r=0.22$, $P=0.003)$; the scale was not associated with the SCI (distress). Cronbach's alpha for the combined English and French sample was $\alpha=0.96$.

\section{Discussion}

This paper describes the preliminary validation of a short measure for physician support of SSE among patients already diagnosed with melanoma, who were recruited from an outpatient secondary care clinic. Factor analyses with English, French, and combined samples suggested that the nine items represent a single factor. High inter-items correlations were observed between items assessing recommendations for SSE (item \#4) and recommendations for regular SSEs (item \#5) and between recommendations for regular SSE (item \#5) and recommendations for SSE of the entire body (item \#6). These correlations are not unusual, given the overlapping content of the respective items. However, removing item \#5 did not result in improved fit for the overall model. The high correlations between these items could be partially explained by the lack of evidence on the optimal frequency of SSE: while there are currently no randomized controlled trials assessing the impact of SSE on melanoma early detection or mortality (Coroiu et al., in preparation), frequency of SSE included in clinical care guidelines range from monthly to yearly (Watts et al., 2015). This is likely to influence the consistency of physician recommendations for SSE, which are reflected in patients' self-reports of the recommendations. The physician support of SSE scale had moderate positive associations with measures of perceived support from HCP, previous recommendation and demonstration of SSE and previous skin examination by HCP, and intentions to perform SSE, 
Table 2 Factor loadings, confidence intervals (Cl), means (and standard deviations), and Pearson's inter-item (and corrected item-total) correlations

\begin{tabular}{|c|c|c|c|c|c|c|c|c|c|c|c|c|}
\hline Items $^{a}$ & $\begin{array}{l}\text { Factor } \\
\text { loading }\end{array}$ & $95 \% \mathrm{Cl}$ & $M(\mathrm{SD})$ & 1 & 2 & 3 & 4 & 5 & 6 & 7 & 8 & 9 \\
\hline 1. It seems important to my physician that I do SSE & 0.93 & {$[0.89,0.96]$} & $2.04(1.18)$ & - & & & & & & & & \\
\hline $\begin{array}{l}\text { 2. My physician explains how to recognize suspicious } \\
\text { changes on my skin }\end{array}$ & 0.88 & {$[0.83,0.93]$} & $1.42(1.26)$ & 0.67 & - & & & & & & & \\
\hline $\begin{array}{l}\text { 3. My physician has pointed out a lesion(s) that I should } \\
\text { keep an eye on }\end{array}$ & 0.79 & {$[0.71,0.86]$} & $1.25(1.33)$ & 0.51 & 0.70 & - & & & & & & \\
\hline $\begin{array}{l}\text { 4. My physician has recommended that I do skin self- } \\
\text { exams }\end{array}$ & 0.98 & {$[0.97,0.99]$} & $1.77(1.30)$ & 0.83 & 0.74 & 0.62 & - & & & & & \\
\hline $\begin{array}{l}\text { 5. My physician has recommended that I examine my } \\
\text { skin regularly }\end{array}$ & 0.99 & {$[0.98,1.0]$} & $1.73(1.29)$ & 0.81 & 0.73 & 0.60 & 0.92 & - & & & & \\
\hline $\begin{array}{l}\text { 6. My physician has recommended that I examine the } \\
\text { skin of my whole body }\end{array}$ & 0.97 & {$[0.96,0.99]$} & $1.62(1.33)$ & 0.75 & 0.69 & 0.54 & 0.86 & 0.90 & - & & & \\
\hline $\begin{array}{l}\text { 7. My physician has recommended that someone help } \\
\text { me to self-examine hard-to-see parts of my body }\end{array}$ & 0.93 & {$[0.9,0.96]$} & $1.36(1.33)$ & 0.64 & 0.68 & 0.52 & 0.77 & 0.76 & 0.82 & - & & \\
\hline $\begin{array}{l}\text { 8. My physician has asked me if I have questions or } \\
\text { concerns about examining my skin }\end{array}$ & 0.89 & {$[0.85,0.94]$} & $1.36(1.30)$ & 0.62 & 0.68 & 0.54 & 0.68 & 0.68 & 0.74 & 0.75 & - & \\
\hline 9. My physician has asked me if I do SSE & 0.95 & {$[0.93,0.97]$} & $1.48(1.32)$ & 0.73 & 0.70 & 0.59 & 0.85 & 0.83 & 0.80 & 0.78 & 0.78 & - \\
\hline Corrected item-total correlations & - & - & - & $(0.80)$ & $(0.80)$ & $(0.65)$ & $(0.65)$ & $(0.91)$ & (0.89) & $(0.83)$ & $(0.78)$ & $(0.88)$ \\
\hline
\end{tabular}

All correlations were significant at $P<0.001$.

द a French items available upon request from main author.

SSE $=$ skin self-examination. 
which lends support for the convergent validity of the measure. The scale was not associated with demographic characteristics or with skin cancer distress, providing evidence for the divergent validity of the physician support for SSE scale. Further, internal consistency reliability of the combined English and French samples was excellent.

This scale was designed primarily as an outcome measure for intervention studies with individuals at high risk for melanoma (and other skin cancers) in order to identify those who have not been instructed to perform SSE or educated about SSE by physicians, and who could benefit from interventions to facilitate SSE uptake. Although the participants recruited in the current study were all secondary care patients, there are other individuals at high risk for whom this scale is appropriate, including first-degree relatives of melanoma patients, survivors of childhood cancers treated with radiation, transplant recipients, for whom clinical care guidelines also recommend SSE (for a review of clinical care guidelines, see Watts et al., 2015). Pending further psychometric evaluation, the items of this scale (English and French versions) could be modified for applicability to other healthcare professionals, including nurses. Given the limited time physicians can allocate to each patient, it might simply be more feasible that the recommendations to check the skin be provided by other professionals on the care team, with nurses being best positioned for this important task.

An important limitation of the current study is the lack of a qualitative component to inform the item development phase of the scale under investigation. While we conducted extensive literature reviews on the topic of physician recommendations for health behaviours, including SSE, and consulted with melanoma prevention experts and clinical dermatologists, we did not conduct interviews or focus groups with patients.

Given the importance of physician recommendations for SSE, that no other scale assessing this construct currently exists, and based on our results, we recommend that the nine-item version of the scale be retained in future research with individuals at high risk for melanoma, for whom SSE is highly applicable. We also recommend that future studies using this scale should focus on further investigating its psychometric properties. Future versions of this scale might specify the optimal frequency of SSE, as more research becomes available, and might incorporate items reflecting recommendations to use aids while performing SSE (eg, mirrors, melanoma pictures, partners); might update the instructions embedded in the scale to include other healthcare professionals, such as nurses; and might add other relevant items, as they become apparent from conversations with patients.

\section{Acknowledgements}

We would like to thank our participants who graciously offered their time to complete our study and the numerous research assistants with the Health Psychology Research Group (HPRG) at McGill who collected data for this study.

\section{Conflicts of Interest}

None.

\section{Financial Support}

The longitudinal study was supported by operating grants from the Fonds de la Recherche en Santé du Québec (FRSQ) and the Canadian Institute for Health Research (CIHR) awarded to Annett Körner, PhD. Adina Coroiu, MA, PhD Candidate is supported by a doctoral training award from CIHR; Chelsea Moran, BA, and Rosalind Garland, BA were supported by master's training awards from CIHR.

\section{Ethical Standards}

The authors assert that all procedures contributing to this work comply with the ethical standards of the relevant national and institutional guidelines on human experimentation and with the Helsinki Declaration of 1975, as revised in 2008. Ethical approval was obtained from the necessary hospital and university research ethics boards.

\section{References}

Bellizzi, K.M., Rowland, J.H., Jeffery, D.D. and Mcneel, T. 2005: Health behaviors of cancer survivors: examining opportunities for cancer control intervention. Journal of Clinical Oncology 23, 8884-893. 
Bentler, P.M. 1990: Comparative fit indexes in structural models. Psychological Bulletin 107, 238-46.

Bradford, P.T., Freedman, D., Goldstein, A.M. and Tucker, M.A. 2010: Increased risk of second primary cancers after a diagnosis of melanoma. Archives of Dermatology 146, 265-72.

Browne, M.W. and Cudeck, R. 1993: Alternative ways of assessing fit. In Bollen, K.A. and Long, J.S., editors, Testing structural equation models. Newbury Park, CA: Sage, 136-62.

Carli, P., De Giorgi, V., Palli, D., Maurichi, A., Mulas, P., Orlandi, C., Imberti, G.L., Stanganelli, I., Soma, P. and Dioguardi, D. 2003: Dermatologist detection and skin self-examination are associated with thinner melanomas: results from a survey of the Italian Multidisciplinary Group on Melanoma. Archives of Dermatology 139, 607-12.

Cohen, J. 1988: Statistical power analysis for the behavioral sciences. Hillsdale, NJ: Lawrence Erlbaum Associates.

Coroiu, A., Moran, C., Kingsland, E., Thombs, B.D. and Körner, A. In preparation: The effect of psycho-educational interventions on health and process outcomes in individuals at increased risk for melanoma: a systematic review of randomized controlled trials (Prospero trial registration CRD42016033765).

Coups, E.J., Manne, S.L., Stapleton, J.L., Tatum, K.L. and Goydos, J.S. 2016: Skin self-examination behaviors among individuals diagnosed with melanoma. Melanoma Research 26, 71-76.

Geller, A.C. 2009: Educational and screening campaigns to reduce deaths from melanoma. Hematology/Oncol Clinics of North America 23, 515-27.

Hu, L. and Bentler, P.M. 1999: Cutoff criteria for fit indexes in covariance structure analysis: conventional criteria versus new alternatives. Structural Equation Modeling: A Multidisciplinary Journal 6, 1-55.

Katalinic, A., Waldmann, A., Weinstock, M.A., Geller, A.C., Eisemann, N., Greinert, R., Volkmer, B. and Breitbart, E. 2012: Does skin cancer screening save lives?: An observational study comparing trends in melanoma mortality in regions with and without screening. Cancer $118,5395-402$.

Kline, R.B. 2005: Principles and practice of structural equation modeling. New York: Guilford Press.

Körner, A., Coroiu, A., Martins, C. and Wang, B. 2013a: Predictors of skin self-examination before and after a melanoma diagnosis: the role of medical advice and patient's level of education. International Archives of Medicine 6, 8.

Körner, A., Drapeau, M., Thombs, B.D., Rosberger, Z., Wang, B., Khanna, M., Spatz, A., Coroiu, A., Garland, R. and Batist, G. 2013b: Barriers and facilitators of adherence to medical advice on skin self-examination during melanoma follow-up care. BMC Dermatology 13, 1-10.

Loescher, L.J., Harris, R.B., Lim, K.H. and Su, Y. 2006: Thorough skin self-examination in patients with melanoma. Oncology Nursing Forum 33, 633-37.
Manne, S., Fasanella, N., Connors, J., Floyd, B., Wang, H. and Lessin, S. 2004: Sun protection and skin surveillance practices among relatives of patients with malignant melanoma: prevalence and predictors. Preventive Medicine 39, 36-47.

Manne, S. and Lessin, S. 2006: Prevalence and correlates of sun protection and skin self-examination practices among cutaneous malignant melanoma survivors. Journal of Behavioral Medicine 29, 419-34.

Marciano, N.J., Merlin, T.L., Bessen, T. and Street, J.M. 2014: To what extent are current guidelines for cutaneous melanoma follow up based on scientific evidence? International Journal of Clinical Practice 68, 761-70.

Mujumdar, U.J., Hay, J.L., Monroe-Hinds, Y.C., Hummer, A.J., Begg, C.B., Wilcox, H.B., Oliveria, S.A. and Berwick, M. 2009: Sun protection and skin self-examination in melanoma survivors. Psycho-Oncology 18, 1106-115.

Muthén, L.K. and Muthén, B.O. 2007: Statistical analysis with latent variables using Mplus. Los Angeles, CA: Muthén \& Muthén.

Pollitt, R.A., Geller, A.C., Brooks, D.R., Johnson, T.M., Park, E.R. and Swetter, S.M. 2009: Efficacy of skin self-examination practices for early melanoma detection. Cancer Epidemiology Biomarkers \& Prevention 18, 3018-23.

Reise, S.P., Widaman, K.F. and Pugh, R.H. 1993: Confirmatory factor analysis and item response theory: two approaches for exploring measurement invariance. Psychological Bulletin 114, 552-66.

Rhee, J.S., Matthews, B.A., Neuburg, M., Burzynski, M. and Nattinger, A.B. 2005: Creation of a quality of life instrument for nonmelanoma skin cancer patients. The Laryngoscope 115, 1178-185.

Robinson, J.K., Wayne, J.D., Martini, M.C., Hultgren, B.A., Mallett, K.A. and Turrisi, R. 2016: Early detection of new melanomas by patients with melanoma and their partners using a structured skin self-examination skills training intervention: a randomized clinical trial. JAMA dermatology 152, 979-85.

Steiger, J.H. 1990: Structural model evaluation and modification: an interval estimation approach. Multivariate Behavioral Research 25, 173-80.

Swetter, S.M., Pollitt, R.A., Johnson, T.M., Brooks, D.R. and Geller, A.C. 2012: Behavioral determinants of successful early melanoma detection. Cancer 118, 3725-3734.

Tsao, H., Rogers, G.S. and Sober, A.J. 1998: An estimate of the annual direct cost of treating cutaneous melanoma. Journal of the American Academy of Dermatology 38, 669-680.

Tucker, L.R. and Lewis, C. 1973: A reliability coefficient for maximum likelihood factor analysis. Psychometrika 38, 1-10.

Wang, B. 2007: Montreal University Health Centre (MUHC) Melanoma Team: guidelines of care for cutaneous melanoma. Montréal: Québec. 


\section{Adina Coroiu et al.}

Watts, C.G., Dieng, M., Morton, R.L., Mann, G.J., Menzies, S.W. and Cust, A.E. 2015: Clinical practice guidelines for identification, screening and follow-up of individuals at high risk of primary cutaneous melanoma: a systematic review. British Journal of Dermatology 172, 33-47.

Williams, G.C., Grow, V.M., Freedman, Z.R., Ryan, R.M. and

Deci, E.L. 1996: Motivational predictors of weight loss and weight-loss maintenance. Journal of Personality and Social Psychology 70, 115-26.
Zschocke, I., Augustin, M. and Muthny, F.A. 1996: Coping in patients with malignant melanoma at different stages of the illness trajectory (German). Psychomedicine 8, 83-88.

Zschocke, I., Rhein, J., Grimme, H., Stein, B., Muthny, F.A. and Augustin, M. 2000: Self-examination of patients with malignant melanoma in the aftercare: relevance of psychosocial factors and instructions by the physicians. Dermatology and Psychosomatics 1, 8-14. 